\title{
Improving outcomes and decreasing costs of neurological diseases: Mind the gap
}

Neurological disorders are the leading cause of disability-adjusted life-years (DALYs) and the second leading cause of deaths. The four main contributors to neurological DALYs are stroke, migraine, dementia, and meningitis [1]. Global and regional actions to decrease this burden are urgently needed. To appropriately set priorities and allocate resources for such actions, political leaders need to be informed by epidemiological and health economic studies.

In a timely and commendable initiative, the European Brain Council (EBC) launched the Value of Treatment (VoT) for Brain Disorders project, with a focus on the benefits of improved healthcare, the identification of treatment gaps along the patient care pathway, and the estimation of associated cost savings. The first publications resulting from this project appeared recently in the European Journal of Neurology, dealing with four disorders: stroke, Parkinson's disease (PD), normal-pressure hydrocephalus (NPH) and restless legs syndrome (RLS) [2-6].

Barral et al. [2] reported on a cross-sectional survey on loss of productivity and informal care costs related to ischemic stroke in a regional population-based cohort study. Most patients were retired or unemployed, and the response rate was $42 \%$. The mean cost of productivity losses was estimated at $€ 7589 \pm € 12,305$ per patient in the first post-stroke year, with $5.4 \%, 71.2 \%$, and $23.4 \%$ of these being attributed to presenteeism (decreased productivity at work), absenteeism, and leisure time, respectively. The annual mean total cost of informal care was $€ 10,635$ per caregiver. This study illustrates how informal care and productivity losses of patients with ischemic stroke represent a significant economic burden for society, comparable to direct costs. These costs should be included in economic evaluations of stroke.

An analysis of the patient pathway for ischemic stroke [3] identified atrial fibrillation treatment, access to rehabilitation and implementation of Comprehensive Stroke Services as unmet needs. Availability of acute endovascular treatment was analysed as a component of Comprehensive Stroke Services. Full implementation of these services was associated with a $9.8 \%$ absolute reduction in the risk of dependency or death and was cost-effective. The results of this study are relatively robust and are a valid argument for launching new Comprehensive Stroke Centres and scaling up some existing primary stroke centres.

Parkinson's disease affects approximately two in every 1000 people in Europe. The economic evaluation of PD management by Dodel et al. [4] focused on early initiation of treatment, best treatment for advanced disease (deep brain stimulation and best medical treatment, including pump therapies), and adherence to treatment. Filling the current gaps for these interventions was found to be cost-effective for all of them. Achieving the target adherence to treatment would generate the highest cost savings (€239,000-€576,000 in Germany). Greater savings will be obtained when a disease-modifying drug becomes available, because $\mathrm{PD}$ is already quite advanced when the first symptoms of became apparent.

Idiopathic NPH is an important cause of gait, balance, cognitive, and sphincter disturbances in elderly people [7]. More than $25 \%$ of such patients show gait improvement after shunting, but only $50 \%$ to $60 \%$ improve in cognition and bladder function [8] Within 30 days, $25 \%$ of shunted patients need hospital admission or experience a complication, including intracranial bleeding [6]. The proportion of patients receiving shunting is currently low, probably due to a lack of awareness and fear of complications among patients and referring doctors. These treatment gasps were identified in a care pathway analysis by Tinelli et al. [5], who also performed a cost-effectiveness analysis of delivering shunt surgery to patients with idiopathic NPH. They compared moving from the $25 \%$ of patients who undergo surgery nowadays to an ambitious target of $90 \%$. Timely and adequate shunting was cost-effective, with a cost per QALY saved of $€ 20,202$ at 5 years and $€ 35,128$ at 15 years, although 15 years is an optimistic length of survival, considering the median age and comorbidities of idiopathic NPH patients [7,9].

For many people with RLS, symptoms are fortunately mild or intermittent. Diagnosis is often delayed as symptoms are attributed to other conditions. Trenkwalder et al. [6] estimated that the yearly cost of RLS for Germany, France and Italy, for an RLS prevalence of 1.6 , could reach $€ 2,018,868 \mathrm{~m}$. Three treatment gaps were identified: delayed diagnosis; insufficient response; and augmentation. Economic savings when closing the three treatment gaps, were $€ 1600$ to $€ 33,300$ over a period of 54 years for delayed diagnosis, $€ 3600$ to $€ 7800$ for an 11-year time horizon and $€ 8900$ to $€ 36,000$ for a 4-year time horizon per patient case, for insufficient response and augmentation, respectively. Given the high prevalence of RLS, the authors stress the importance of education for all subspecialities involved in RLS patient care and the public. This should also include general practice, as the first medical encounter for many patients with RLS will be their general practitioner. 
Health economic and patient pathway analyses still face important methodological limitations, in particular due to the low quality of evidence describing patient itinerary and supporting (or not) the value of each of its steps. For most neurological diseases, there is, in general, high-quality evidence on which to base decisions to perform or avoid therapeutic interventions. Unfortunately, this is not always the case for diagnostic procedures and processes of care, for which we often have to rely only on expert opinions. High-quality observational studies and pragmatic trials with generalizable results are needed to improve the inputs of health economic studies. The analytic process for these studies implies a "single chronic disease" approach. However, comorbidities may make long-term scenarios unrealistic, as they compete for the unfavorable outcomes and may offset gains obtained by earlier and better diagnosis and management.

Increased costs of prolonged later phases of chronic diseases and upfront payments to improve facilities and processes of care are regarded suspiciously by politicians. Their transient years in office are usually shorter than the latency time between intervention and the point when health gains are obtained. The responsibility will fall on healthcare professionals and scientists to influence the public and politicians in order to translate the evidence provided by the five studies resulting from the EBC project into policies to improve the care and outcomes of individuals with chronic neurological disorders [10].

\section{CONFLICT OF INTEREST}

The author declares no financial or other conflicts of interest.

$$
\text { José M Ferro }{ }^{1,2,3}
$$

${ }^{1}$ Serviço de Neurologia, Department of Neurosciences and Mental Health, Hospital de Santa Maria - CHULN, Lisboa, Portugal

${ }^{2}$ Faculdade de Medicina, Universidade de Lisboa, Lisboa,

Portugal
${ }^{3}$ Faculdade de Medicina, Instituto de Medicina Molecular, Universidade de Lisboa, Lisboa, Portugal Email:jmferro@medicina.ulisboa.pt

\section{ORCID}

José M Ferro (D) https://orcid.org/0000-0002-2343-9097

\section{REFERENCES}

1. GBD 2016 Neurology Collaborators. Global, regional, and national burden of neurological disorders, 1990-2016: a systematic analysis for the Global Burden of Disease Study 2016. Lancet Neurol. 2019;18(5):459-480.

2. Alastair W, Fonseca AC, Elvind B, et al. Value of treatment by comprehensive stroke services for the reduction of critical gaps in acute stroke care in Europe. Eur J Neurol. 2020.

3. Barral M, Rabier H, Termoz A, et al. Patients' productivity losses and informal care costs related to ischemic stroke: a French population-based study. Eur J Neurol. 2020.

4. Dodel R, Tinelli M, Deuschl G, et al. The economic benefit of a timely, adequate, and adherent Parkinson's disease treatment. The value of treatment project 2. Eur J Neurol. 2020.

5. Tinelli M, Guldemond N, Kehler U. Idiopathic Normal Pressure Hydrocephalus (iNPH): the cost-effectiveness of delivering timely and adequate treatment in Germany. Eur J Neurol. 2020.

6. Trenkwalder C, Sakkas G, Dauvilliers Y, et al. Socio-economic impact of Restless Legs Syndrome and inadequate RLS management across European settings. Eur J Neurol. 2020.

7. Zaccaria V, Bacigalupo I, Gervasi G, et al. A systematic review on the epidemiology of normal pressure hydrocephalus. Acta Neurol Scand. 2020;141(2):101-114.

8. Giordan E, Palandri G, Lanzino G, et al. Outcomes and complications of different surgical treatments for idiopathic normal pressure hydrocephalus: a systematic review and meta-analysis. J Neurosurg. 2019;131:1024-1036.

9. Nadel JL, Wilkinson DA, Linzey JR, et al. Thirty-day hospital readmission and surgical complication rates for shunting in normal pressure hydrocephalus: a large national database analysis. Neurosurgery. 2020;86:843-850.

10. Feigin VL, Vos T, Nichols E, et al. The global burden of neurological disorders: translating evidence into policy. Lancet Neurol. 2020;19(3):255-265. 